\title{
Finger usage and arithmetic in adults with math difficulties: evidence from a case report
}

\author{
Liane Kaufmann ${ }^{1 *}$, Silvia Pixner ${ }^{1}$ and Silke M. Göbel ${ }^{2}$ \\ Institute of Applied Psychology, University for Health Sciences, Medical Informatics and Technology, Hall in Tyrol, Austria \\ 2 Department of Psychology, University of York, York, England \\ *Correspondence: liane.kaufmann@umit.at
}

\section{INTRODUCTION}

The role of finger usage upon learning arithmetic has received increasing interest from various disciplines. In this opinion paper, we would like to emphasize that finger usage in calculation is not a unitary phenomenon. Rather, we propose two different types of finger usage: First, in many countries and independent of the number system in use, typically developing children use fingers as important transitory (and intuitive) tools to represent small quantities (Butterworth, 1999; Bender and Beller, 2011). According to Di Luca and Pesenti (2011), finger counting habits are needed to build, acquire, and access mental number representations, the building blocks for semantic number knowledge. Second, developmentally inappropriate finger usage of children with math difficulties (MD) reflects their persistent need to apply back-up strategies to compensate for deficient or lacking number representations (e.g., Brissaud, 1992; Kaufmann, 2002; Wright et al., 2002). Here, we will focus on the second type of finger usage (i.e., persistent finger usage in individuals with MD) and we argue that (a) finger usage in arithmetic is not restricted to children; and (b) finger-based calculation strategies utilized by adults with MD reflect immature calculation strategies that are comparable to those displayed by affected children.

\section{FINGER USAGE IN ADULTS: A CASE REPORT}

In contrast to the extensive literature on children's finger usage, respective studies of adults are lacking. In the following, we will present data of a bright young adult (RM) who experiences severe difficulties with arithmetic. RM is a psychology undergraduate student at the end of her second year. RM's intelligence, reading, and spelling skills as well as her working memory resources are average (short form of the Wechsler Adult Intelligence Scale full-scale IQ 108; Wide Range Achievement Test (WRAT3) read- ing and spelling standard scores (SS) 119 and 101, respectively; digit and spatial span forward/backward scaled scores 12 and 10, respectively). RM's arithmetic skills, however, are weak (WRAT3 arithmetic SS 80).

RM's performance for simple number facts was atypical and maladaptive. Number facts are single-digit mental calculations and are generally encoded and retrieved from long-term memory (Ashcraft, 1992). On the contrary, many children with MD find it hard to store and/or retrieve number facts, despite average non-numerical memory and intact procedural arithmetic skills (for a respective single case study, see Kaufmann, 2002).

RM's performance for single-digit number facts was highly deficient albeit very accurate (addition 100\%, subtraction 100\%, multiplication $99 \%$ correct). Her solution strategies were highly unusual for an adult: Across all three operations, RM used procedural strategies (PS) considerably more often than direct fact retrieval (FR; Table 1). For nearly all problems where she used PS she also used finger counting. Consequently, RM's processing times were considerably longer on problems that were solved procedurally. In addition and subtraction, the most dominant procedural strategy was "counting up/down by one," in multiplication "counting up by ones and twos," and a combination of "retrieval of a 5-table with subsequent counting up/down."

It is interesting to note that RM utilized her 5-table knowledge to solve multiplication facts by combining FR with a counting up strategy (for an example, see below). Nonetheless, RM was not able to utilize (parts of a) 2-table or 10-table knowledge similarly [as most children with and without MD would; e.g., $4 * 3=(2 * 3)+(2 * 3)$ or $9 * 3=(10 * 3)-(1 * 3)]$

A typical example of RM's solution strategy for multiplication facts: upon solving the problem $7 * 8$, she calculated $5 * 8=40$ (using 5-table knowledge), then added two sets of eight by counting up. Thereby, RM had to add the two sets of eight by the time consuming strategy of counting up by ones $(40+1+1+1+1+1+1+1+1=48$; then $48+1+1+1+1+1+1+1+1=56)$. For both sets (as well as for all other problems solved by finger counting) she started the counting procedure by bending first her left-hand thumb, then the index, middle, ring and little finger and continued the counting process with her right-hand thumb. The solution time for this problem was $18.5 \mathrm{~s}$. Notably, it took RM $39.6 \mathrm{~s}$ to solve the reversed problem presented several trials earlier. This complicated back-up strategy demonstrates clearly that RM has excellent conceptual and procedural arithmetic knowledge, but due to her patchy fact knowledge and severe difficulty to perform carry procedures, her solution strategies (while errorless) become very time consuming.

In essence, RM's fact knowledge was restricted to rule-based number facts $(n+0, n+1, n * 0, n * 1)$. Out of 32 addition facts that were solved by direct memory retrieval (total $n=100$ ), 25 were classified as so-called "rule-based facts" (15 number facts were of the type $n+0,10$ further number facts were of the type $n+1)$. Out of 22 subtraction facts solved by retrieval (total $n=42$ ), 15 were rule-based facts. Out of 54 multiplication problems (total $n=100$ ) solved by direct memory retrieval, 19 were $n * 0,17 n * 1$, and $15 n * 5$ problems.

\section{SYNOPSIS}

To summarize, we argue that (i) finger usage in calculation is not restricted to children; and (ii) the qualitative analysis of finger use and its function (in children and adults) may provide important indirect insights into the organization of number fact representations. It is plausible that children and adults with MD who use finger-based calculation strategies develop and employ their finger counting strategies to a much higher and more complex level. 
Table 1 | Solution strategies employed by RM upon solving number facts (single-digit problems). Procedural strategies (PS) are differentiated from fact retrieval (FR). For PS the various counting strategies employed by RM are described. Reported are frequency of use in $\%$ and mean reaction times (RT) with standard deviations (SD) where applicable.

\section{Solution strategy}

Frequency of use [correct out of maximum possible] (mean RT/SD)

\begin{tabular}{llll}
\cline { 2 - 3 } & $\begin{array}{l}\text { Addition } \\
(\boldsymbol{n}=\mathbf{1 0 0})\end{array}$ & $\begin{array}{l}\text { Subtraction } \\
(\boldsymbol{n}=\mathbf{4 2})\end{array}$ & $\begin{array}{l}\text { Multiplication } \\
(\boldsymbol{n}=100)\end{array}$ \\
\hline FR overall & $\begin{array}{l}32.0 \%[32 / 100] \\
(1.8 \mathrm{~s} / 0.8 \mathrm{~s})\end{array}$ & $\begin{array}{l}52.4 \%[22 / 42] \\
(1.5 \mathrm{~s} / 0.6 \mathrm{~s})\end{array}$ & $\begin{array}{l}54.0 \%[54 / 100] \\
(1.6 \mathrm{~s} / 0.9 \mathrm{~s})\end{array}$ \\
PS overall & $68.0 \%[68 / 100]$ & $47.6 \%[20 / 42]$ & $46.0 \%[46 / 100]$ \\
& $(3.1 \mathrm{~s} / 1.3 \mathrm{~s})$ & $(4.7 \mathrm{~s} / 2.1 \mathrm{~s})$ & $(12.0 \mathrm{~s} / 8.9 \mathrm{~s})$ \\
PS: FR after change & $11.8 \%[8 / 68]$ & n.a. & n.a. \\
of operands & $(2.0 \mathrm{~s} / 0.6 \mathrm{~s})$ & & $13.0 \%[6 / 46]$ \\
PS: Counting up/down & $88.2 \%[60 / 68]$ & $95 \%[19 / 20]$ & $(10.1 \mathrm{~s} / 6.6 \mathrm{~s})$ \\
by ones & $(3.2 \mathrm{~s} / 1.3 \mathrm{~s})$ & $(4.8 \mathrm{~s} / 2.2 \mathrm{~s})$ & $23.9 \%[11 / 46]$ \\
PS: Counting up in & n.a. & n.a. & $(3.8 \mathrm{~s} / 2.3 \mathrm{~s})$ \\
twos & & & $63.0 \%[29 / 46]^{*}$ \\
PS: Combined FR AND & n.a. & $5.0 \%[1 / 20]$ & $(16.2 \mathrm{~s} / 8.6 \mathrm{~s})$ \\
count up/count down & & $(3.3 \mathrm{~s})$ & \\
\hline
\end{tabular}

Abbreviation: n.a., not applicable.

*Notes: Among the 29 problems solved by a combination of FR and counting up, 27 were $n * 5$ problems, one was a $n * 6$ problem and one was solved by a combination of retrieving a 5-table and subtracting one operand from this result $(6 * 4=6 * 5-6)$.

For addition and multiplication number facts, all problems with operands from 0 to 9 were presented (summing up to 100 problems per operation). Each problem was presented only once, reversed digit order was considered a separate problem $(2+3,3+2)$. In addition and multiplication, 36 out of the 100 problems were so-called "rule-based number facts" (i.e., problems containing either a 0 or a 1). For subtraction, 42 single-digit problems with minuends and subtrahends between 9 and 1 were presented (please note that due to experimental failure two problems (8-2 and 4-1) were not administered).

RM demonstrated preserved rule-based fact knowledge but had severe difficulties to solve the remaining number facts. This observation further supports the notion that rule-based number facts are stored and encoded differently (Ashcraft, 1992; for evidence from brain imaging studies, see Jost et al., 2004, 2009). Notably, RM was not able to directly retrieve multiplication facts other than the 5-tables. RM's prolonged and extensive finger use might have specifically facilitated the storage and access of the 5 -tables because fingers of one hand sum up to five (Domahs et al., 2008). Importantly, some of the solution strategies employed by RM (especially the extensive use of 5-tables in combination with counting up or down) reflect excellent conceptual and procedural arithmetical knowledge. With respect to acquired calculation disorders as observed in neurological patients that have sustained cerebral injury to number-relevant brain regions, previous case studies have shown that conceptual arithmetical knowledge (i) may be dissociated from other aspects of numerical cognition, and (ii) may be effectively used to bypass deficient number fact knowledge (Hittmair-Delazer et al., 1994; Delazer and Benke, 1997). Furthermore, in the developmental literature, the close interplay between conceptual and procedural knowledge has been repeatedly emphasized (e.g., Rittle-Johnson et al., 2001). The case of a developmental MD in adulthood reported here corroborates both the neuropsychological and the developmental literature because it shows that conceptual arithmetical knowledge - in tandem with procedural knowledge - constitutes a valuable tool for bypassing impaired or patchy number fact knowledge. Thus, conceptual arithmetical knowledge should be assigned a key role in developmental and adult calculation models alike. An open question is whether RM's mental number representations are deficient per se (storage deficit) or whether they are difficult to access. Importantly, finger usage while solving number facts does neither indicate the absence or presence of number fact representations nor does it enable us to differentiate between storage and access problems. Instead, systematic investigations targeted at examining interference, consistency and problem size effects may aid us in disentangling storage and access deficits in numerical cognition research (Kaufmann et al., 2004). Though it could be argued that the usage of elaborate strategies instead of fact retrieval shows a good understanding of the number system and relations between operations, the characteristic feature of the finger use displayed by RM reflects a highly immature and inflexible use of fingers as an external token system. Within the developmental framework proposed by Kucian and Kaufmann (2009) RM's counting behavior suggests that the developmental shift from concrete and notation-specific to a more abstract and notation-independent number representation might not have taken place yet.

Overall, this case study shows that (i) finger-based calculation strategies might accompany MD in adults; and (ii) immature calculation strategies may persist despite excellent procedural arithmetic skills and average working memory resources. Nonetheless, RM's persistent finger usage upon solving simple number facts does not allow us to disentangle storage from access problems. A further unresolved issue concerns the question whether in RM the excessive use of finger counting reflects a back-up strategy employed to circumvent poor math skills or whether RM's overly reliance on finger counting might have had detrimental effects on early math development (see Moeller et al., 2011). Though we believe that the finger counting observed in RM most likely reflects a strategy to deal with $\mathrm{MD}$, the latter assumption remains speculative thus far. As a final note we would like to draw the reader's attention to the fact that though average calculating adults do not typically use overt finger counting strategies, their calculation performance may reveal some reliance on finger-based number representations (Klein et al., 2011). However interestingly, with respect to acquired calculation disorders (evolving as a sequence of traumatic brain injury) the current litera- 
ture does not report the use of overt finger counting behavior as a back-up strategy to solve number fact retrieval (e.g., HittmairDelazer et al., 1994; Zaunmüller et al., 2009). A limitation of the present study is the lack of information about RM's early math development which possibly could aid us in disentangling the nature of the excessive finger counting displayed by RM (i.e., finger counting reflecting a strategy to circumvent poor math skills versus finger counting fostering poor math skills by hampering the adoption of mature calculation strategies). Future studies are clearly needed to investigate finger-based calculation strategies in adults with MD in detail and to identify and characterize potential commonalities as well as divergences with (initial and prolonged) finger usage during children's acquisition of arithmetic skills.

\section{ACKNOWLEDGMENT}

We would like to thank RM for her participation. Supported by the Anniversary Fund of the Austrian National Bank (grant number 13454 to Liane Kaufmann) and the Departmental IPR Fund, University of York (Silke M. Göbel).

\section{REFERENCES}

Ashcraft, M.H. (1992). Cognitive psychology and simple arithmetic: a review and summary of new directions. Cognition 44, 75-106.
Bender, A., and Beller, S. (2011). Nature and culture of finger counting: diversity and representational analysis of an embodied cognitive tool. Front. Cogn. 2:256. doi: 10.3389/fpsyg.2011.00256

Brissaud, R. (1992). "A toll for number construction: finger symbol sets," in Pathways to Number: Children's Developing Numerical Abilities, eds J. Bideaud, C. Meljac, and L.-P. Fischer. (Hillsdale, NJ: Lawrence Erlbaum).

Butterworth, B. (1999). The Mathematical Brain.London: McMillan.

Delazer, M., and Benke, T. (1997). Arithmetic facts without meaning. Cortex 33, 697-710.

Di Luca, A., and Pesenti, M. (in press/2011). Fingercounting representations: more than just another symbolic code? Front. Cogn. 2.

Domahs, F., Krinzinger, H., and Willmes, K. (2008). Mind the gap between both hands: evidence for internal finger-based number representations in children's mental calculation. Cortex 44, 359-367.

Hittmair-Delazer, M., Semenza, C., and Denes, G. (1994). Concepts and facts in calculation. Brain $117,715-728$.

Jost, K., Beinhoff, U., Hennighausen, E., and Rösler, F. (2004). Facts, rules, and strategies in singledigit multiplication: evidence from event-related brain potentials. Brain Res. Cogn. Brain. Res. 20, 183-193.

Jost, K., Khader, P., Burke, M., Bien, S., and Rösler, F. (2009). Dissociating the solution processes of small, large, and zero multiplications by means of fMRI. Neuroimage 46, 308-318.

Kaufmann, L. (2002). More evidence for the role of the central executive in retrieving arithmetic facts - a case study of severe developmental dyscalculia.J. Clin. Exp. Neuropsychol. 24, 302-310.

Kaufmann, L., Lochy, A., Drexler, A., and Semenza, C. (2004). Deficient arithmetic fact retrieval - storage or access problem? A case study. Neuropsychologia $42,482-496$.
Klein, E., Moeller, K., Willmes, K., Nuerk, H.-C., and Domahs, F. (2011). The influence of implicit handbased representations on mental arithmetic. Front. Cogn. 2:197. doi: 10.3389/fpsyg.2011.00197

Kucian, K., and Kaufmann, L. (2009). A developmental model of number representations. Behav. Brain Sci. 32, 340-341.

Moeller, K., Martignon, L., Wessolowski, S., Engel, J., and Nuerk, H.-C. (in press/2011). Effects of finger counting on numerical development - a review of neurocognitive arguments and those from mathematics education. Front. Cogn. 2.

Rittle-Johnson, B., Siegler, R. S., and Alibali, M. W. (2001). Developing conceptual understanding and procedural skill in mathematics: an iterative process. J. Educ. Psychol. 93, 346-362.

Wright, R. J., Martland, J., Stafford, A. K., and Stanger, G. (2002). Teaching Number. Advancing Children's Skills and Strategies, 2nd Edn. London: Paul Chapman Publishing Ltd.

Zaunmüller, L., Domahs, F., Dressel, K., Lonnemann, J., Klein, E., Ischebeck, A., and Willmes, K. (2009). Rehabilitation of arithmetic fact retrieval via extensive practice: a combined fMRI and behavioral case-study. Neuropsychol. Rehabil. 19, 422-443.

Received: 24 June 2011; accepted: 13 September 2011; published online: 04 October 2011.

Citation: Kaufmann L, Pixner S and Göbel SM (2011) Finger usage and arithmetic in adults with math difficulties: evidence from a case report. Front. Psychology 2:254. doi: 10.3389/fpsyg.2011.00254

This article was submitted to Frontiers in Cognition, a specialty of Frontiers in Psychology.

Copyright $\odot 2011$ Kaufmann, Pixner and Göbel. This is an open-access article subject to a non-exclusive license between the authors and Frontiers Media SA, which permits use, distribution and reproduction in other forums, provided the original authors and source are credited and other Frontiers conditions are complied with. 